\title{
Impact damage prediction in carbon fiber-reinforced laminated composite using the matrix-reinforced mixing theory
}

\author{
Marco A. Pérez ${ }^{\mathrm{a}, \mathrm{d}, *}$, Xavier Martínez $^{\mathrm{b}, \mathrm{e}}$, Sergio Oller $^{\text {b,d }}{ }^{\text {, Lluís Gil }}{ }^{\mathrm{a}, \mathrm{d}}$, Fernando Rastellini ${ }^{\mathrm{b}, \mathrm{c}}$, Fernando Flores ${ }^{\mathrm{b}, \mathrm{f}}$ \\ a Laboratory for the Technological Innovation of Structures and Materials (LITEM), Colon 11, TR45, Terrassa, 08222 Barcelona, Spain \\ ${ }^{\mathrm{b}}$ International Center for Numerical Methods in Engineering (CIMNE), Gran Capità s/n, Edifici C1, 08034 Barcelona, Spain \\ ${ }^{\mathrm{C}}$ Quantech ATZ S.A., Gran Capità 24, Edifici Nexus, 08034 Barcelona, Spain \\ ${ }^{\mathrm{d}}$ Departament de Resistència de Materials i Estructures a l'Enginyeria, Universitat Politècnica de Catalunya - BarcelonaTech, Spain \\ ${ }^{\text {e }}$ Departament de Ciència i Enginyeria Nàutiques, Universitat Politècnica de Catalunya - BarcelonaTech, Spain \\ ${ }^{\mathrm{f}}$ Departamento de Estructuras, Universidad Nacional de Córdoba, C.C. 916, Córdoba 5000, Argentina
}

\section{A R T I C L E I N F O}

\section{Article history:}

Available online 22 April 2013

\section{Keywords:}

Low-velocity impact

Composite laminates

Damage mechanics

Finite element analysis

\begin{abstract}
A B S T R A C T
The impact damage tolerance of fiber-reinforced laminated composite materials is a source of concern, mainly due to internal induced damage which causes large reductions on the strength and stability of the structure. This paper presents a procedure based on a finite element formulation that can be used to perform numerical predictions of the impact induced internal damage in composite laminates. The procedure is based on simulating the composite performance using a micro-mechanical approach named matrix-reinforced mixing theory, a simplified version of the serial/parallel mixing theory that does not require neither the iterative procedure nor the calculation of the tangent stiffness tensor. The numerical formulation uses continuum mechanics to simulate the phenomenon of initiation and propagation of interlaminar damage with no need to formulate interface elements, resulting in a computationally less demanding formulation. To demonstrate the capability of numerical procedure when applied to a lowvelocity impact problem, numerical results are compared with the experimental ones obtained in a test campaign performed on 44 laminates specimens subjected to an out-of-plane and concentrated impact event, according to ASTM test method. Results are in good agreement with experimental data in terms of delamination onset and the internal spatial distribution of induced damage.
\end{abstract}

(c) 2013 Elsevier Ltd. All rights reserved.

\section{Introduction}

Laminated composites offer decisive advantages when being compared to more traditional materials, particularly in structural applications where weight is a design-limiting factor. However, these materials have also shown a susceptibility to impact damage due to lack of plastic deformation, to the low interlaminar strength and to the laminated construction to reduce the anisotropic nature of the plies. This physical phenomenon limits the widespread application of composite laminates particularly into structures prone to impacts during service [1]. Therefore, in recent decades the topic has become a source of interest and concern that has promoted intensive research.

Impacts are generally classified according to ranges from low to high-velocity impacts. The structural response of composites

* Corresponding author at: Laboratory for the Technological Innovation of Structures and Materials (LITEM), Colon 11 TR45 08222 Terrassa, Barcelona, Spain. Tel.: +34 937398289.

E-mail address: marco.antonio.perez@upc.edu (M.A. Pérez). differs significantly between the two ranges. The response of a laminate subjected to high-velocity impact is dominated by the propagation of compression waves generated in the vicinity of the contact point and transmitted through the thickness, resulting in localized damages $[2,3]$. Despite that low-velocity impacts are simple events, they induce complex effects, compromising the residual bearing capacity of the structural component. Low-velocity impact induced damage is a complex mixture of three main failure modes: matrix cracking, delamination and fiber breakage. Among them, delamination has been found to be the most pernicious damage mode. The situation has been proven critical even when the damaged surface located in the contact area is not representative of the induced damage, causing a collapse under unacceptable compressive load levels [4]. Therefore, to successfully design damage tolerant composite structures, it is necessary to have a reliable analysis tool to aid understanding the complex phenomena that take places, and capable of estimating the consequences of an impact.

The prediction of the impact response and induced damage extended onto composite laminates subjected to low-velocity 
impacts, has been widely treated in literature in terms of analytical, experimental and numerical approaches [5-11]. The analytical formulations proposed are, notwithstanding, restricted to particular cases [12-14]. This is mainly due to the complexity of physical phenomena involved, which require an accurate modeling of dynamics response, contact formulations, failure modes as well as the influence of multiple test conditions. Nevertheless, despite the extensive research effort and significant advances in this area, the goal has not yet been reached. On the other hand, the high-cost and difficult experimentation preclude a completely empirical study for the impact damage prediction. Numerical methodologies based on Finite Element approach are a more flexible alternative, which provide detailed information on the spatial and temporal evolution of damage during the impact event.

Numerous contributions have been made to numerical modeling of impact damage involving composite materials. These studies include impact from low to high velocity, and have been focusing on the prediction of the onset, propagation and extent of damage. A detailed survey of the numerical treatment of composite structures and procedures used nowadays to deal with it can be found in references [15,16]. Different numerical approaches can broadly be classified into four categories [17]: failure criteria, fracture mechanics, plasticity theory and damage mechanics. Failure criteria approaches used polynomial expressions in terms of stress and/or strain, which allow to determine the failure envelopes of the material $[15,18]$. However, their use

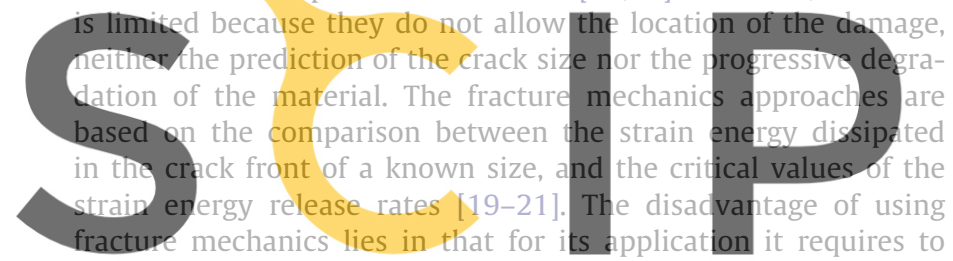
predefine an initial crack region in the numerical model. Regarding the plasticity approach, the key concept is the assumption

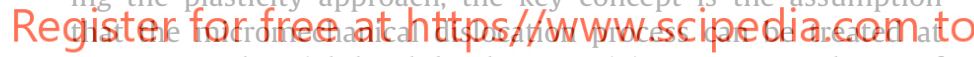
a macromechancial level by homogenizing over a volume of material and described by the plastic strain tensor [22]. However, it should be noted that this approach has been proved to be more suitable for composites exhibiting ductile behavior such as thermoplastics. The continuum damage mechanics approaches [17,23-25] are based on material degradation models that allow simulating the material behavior after the onset of damage. These approaches have proved being successful if they are used with an appropriate failure criteria and fracture mechanics to predict different composite failure modes, including matrix cracking, delamination and fiber breakage [16].

The authors of main works available in the open literature agree on solving the problem of the simulation of quasi-static loading and low velocity impacts events in layered composites, by representing the two processes taking place: first the onset of matrix damage or crack initiation and afterwards its propagation through the laminate, the so-called delamination. The onset of damage can be predicted by several means, such as stress and/or strains failure criteria or fracture mechanics using the energy release rate [15]. However, major efforts have been focused on the treatment of delamination [26]. Among the different strategies for the simulation of delamination process, the most common approaches are the virtual crack closure technique (VCCT) [27] and the cohesive zone models (CZMs) [28-35]. The VCCT calculates energy-release rate, with the assumption that during the crack growth the energy needed to open the crack is the same as the energy needed to close it. The CZM uses cohesive elements placed in the interfaces of the laminae to describe the interlaminar cohesive forces. When damage growth occurs, these cohesive elements open in order to simulate the fracture propagation. It has been shown that both approaches are capable of characterizing the strength of the interface, allowing the simulation of the transient behavior of a laminate into impact event and obtaining successful results of progressive failure of the constituent materials and at the plies interface. However, it should be pointed out that both approaches rely on predefined interfaces that constrain the interlaminar crack path. Consequently, the crack propagation strongly depends on the presence (or absence) of a fracture surface or cohesive zone elements, implying that the crack path is mesh dependent [36]. When the crack path is unknown, fracture surface or cohesive elements must be placed in all the interfaces of the layers of the laminate, which may result, depending on the size of the problem to be solved, computationally unaffordable.

Most of the formulations used nowadays to simulate composite laminates treat them as a single material, with properties inherited from their constituents. This has proven to be inaccurate, as most of the failure mechanisms of composites are because of the micro-structural interaction between its constituents. An example of these interactions can be found in the formulation developed by Martinez et al. in [37] to characterize the compression strength of composites. Therefore, as composites can be considered more as a micro-structural system rather than a material, new formulations and theories are required to predict their mechanical performance accurately.

In contrast with the large number of publications dealing the simulation of low-velocity impact based on failure, fracture, and
plasticity, the present workuses a continuum mechanics approach
to simulate the phenomenon of initiation and propagation fonter-
laminar damage with no need to formulate interface elements that
predefine the crack path. The proposed procedure is based on
simulating the laminate composite by using a micro-mechanical
approach named matrix-reinforced mixing theory/(MR RoIM). This paper enters in the continuity of the work conducted by the authors [38-40]. These works demonstrate that micro-structural

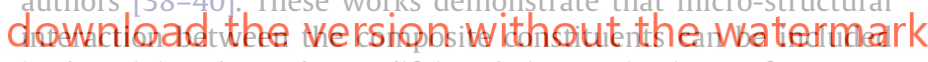

in the mixing theory by modifying their constitutive performance. The model used in the present work consists of a simplified version of the serial/parallel mixing theory (SP RoM), which reduces the computational cost without affecting significantly its simulation performance, in particular, its ability to predict delamination process.

The MR RoM is based on the differentiation and separation of the composite mechanical performance into a parallel and serial component, corresponding the first to the reinforcement direction and the second to the remaining directions. The MR RoM acts as a constitutive equation manager and has proven capable of predicting the composite behavior even when one of its constituents gets into the non-linear range. The validity of the proposed procedure to successfully predict mechanical structural performance of a laminate composite subjected to low velocity impact damage, is assessed with the comparison of the numerical results with those obtained experimentally from the impact ASTM test method.

The following section describes the numerical formulation of the MR RoM, together with the damage model used to predict the matrix mechanical performance. In the next section details and requirements of the experimental impact test procedures are given. In the following, the numerical model developed to investigate the capability of the proposed numerical formulation when applied to a low-velocity impact problem is presented. The numerical results obtained are compared with the experimental ones. Comparisons include predictions of the contact time and the internal spatial distribution of damage. Finally, the conclusions of the study are presented. 


\section{Numerical formulation}

\subsection{Matrix-reinforced mixing theory}

The matrix reinforced mixing theory (MR RoM) has its origin in the serial/parallel mixing theory (SP RoM). ${ }^{1}$ The SP RoM is based on the definition of two different compatibility equations that define the stress equilibrium and establish the strain compatibility between the composite constituent materials. It defines an iso-strain condition in the fiber (parallel) direction and an iso-stress condition in the transversal (serial) direction.

For that purpose, the composite strain tensor $\left({ }^{c} \boldsymbol{\varepsilon}\right)$ is decomposed into its parallel ( ${ }^{C} \boldsymbol{\varepsilon}_{P}$ ) and serial ( ${ }^{C} \boldsymbol{\varepsilon}_{S}$ ) components by using fourth-order projector tensors $\mathbb{P}_{P}$ and $\mathbb{P}_{S}$, respectively, as follows:

${ }^{c} \boldsymbol{\varepsilon}={ }^{c} \boldsymbol{\varepsilon}_{P}+{ }^{c} \boldsymbol{\varepsilon}_{S}$

where

${ }^{c} \mathcal{E}_{P}=\mathbb{P}_{P}:{ }^{c} \mathcal{E}$ and $\quad{ }^{c} \mathcal{E}_{S}=\mathbb{P}_{S}:{ }^{c} \mathcal{E}$

being $\mathbb{P}_{P}=\left(e_{1} \otimes e_{1}\right) \otimes\left(e_{1} \otimes e_{1}\right), \mathbb{P}_{S}=\left(\square-\mathbb{P}_{P}\right), e_{1}$ the vector which defines the parallel behavior (fiber direction), and $\llbracket$ the identity.

In the proposed numerical model, the response of the composite material is considered to be directly related to the volume fractions of its constituent materials, and phases in the composite are assumed to be homogeneously distributed. The equilibrium and compatibility equations describing the interaction between the constituent materials are:

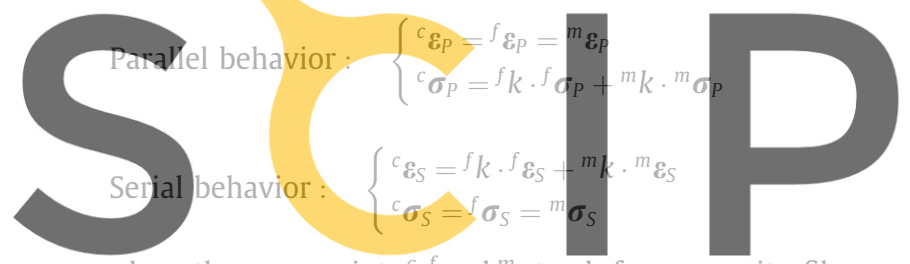

$(4)$

where the superscripts ${ }^{c}$, and $^{m}$ stands for composite, fiber and matrix, respectively and ${ }^{i} k$ is the volume-fraction coefficient of each

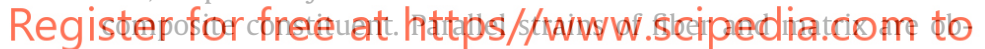
tained straightforward from the composite strains. However, the compatibility equations are not fulfilled so easily in the serial direction, being necessary to make an initial prediction on the fiber or matrix serial strain. Once the strains of both components are known, their stress tensor can be calculated using any given constitutive law. Therefore, it is possible to simulate the composite constituents with any kind of non-linear material behavior, such as damage or plasticity. With this approach, if one of the component materials becomes non-linear, the initial prediction may be inaccurate and it is necessary an iterative procedure to fulfill equations Eqs. (3) and (4).

Given that an explicit time integration scheme evaluates the material constitutive equation at each time step, this procedure may be unfeasible depending on the size of time step and/or the size of the problem. With this scenario, the MR RoM was proposed in [38] as a method computationally less demanding to perform large simulations of composite structures. It states that in multilayer laminates the contribution of fibers to the stiffness and strength in the transverse direction (serial) of a single layer can be neglected, especially if the laminate has several layers oriented in different directions. This assumption leads to the following compatibility equations:

Parallel behavior : $\quad\left\{\begin{array}{l}{ }^{c} \boldsymbol{\varepsilon}_{P}={ }^{f} \boldsymbol{\varepsilon}_{P}={ }^{m} \boldsymbol{\varepsilon}_{P} \\ { }^{c} \boldsymbol{\sigma}_{P}={ }^{f} k \cdot{ }_{k} \boldsymbol{\sigma}_{P}+{ }^{m} \boldsymbol{k} \cdot{ }^{m} \boldsymbol{\sigma}_{P}\end{array}\right.$

\footnotetext{
${ }^{1}$ The formulations used are thoroughly described in references [38,41] and will not be elaborated here except for their main aspects which are briefly described in the following.
}

Serial behavior : $\left\{\begin{array}{l}{ }^{c} \boldsymbol{\varepsilon}_{S}={ }^{m} \boldsymbol{\varepsilon}_{S} \\ { }^{c} \boldsymbol{\sigma}_{S}={ }^{m} \boldsymbol{\sigma}_{S}\end{array}\right.$

Under this assumption, it is noted that both serial and parallel strains of fiber and matrix can be obtained straightforward from the composite strains, avoiding the iterative procedure. As a further consequence, it will not require the evaluation of the tangent stiffness tensor of the composite components, which results in a significant reduction of the computational cost.

As per impact induced damages, the weakening of the matrix due to excessive shear stresses, will result in the inability to develop either longitudinal, transverse or shear stresses. According to the above compatibility equations, when matrix reaches a critical stress state and its strength and stiffness is reduced, the whole composite stress and stiffness will be also reduced in the serial direction. If shear stresses are defined with the serial condition, the composite shear strength and stiffness will be reduced as occurs in a delaminated composite. It has been already proved that this is equivalent to a delamination failure mode [38].

\subsection{Matrix damage model}

Material degradation in a continuum solid due to fracture process can be simulated with a damage formulation. This formulation takes into account the reduction of the effective area of the material, reducing its stiffness properties. The procedure proposed in this work to simulate impact uses a damage constitutive law to

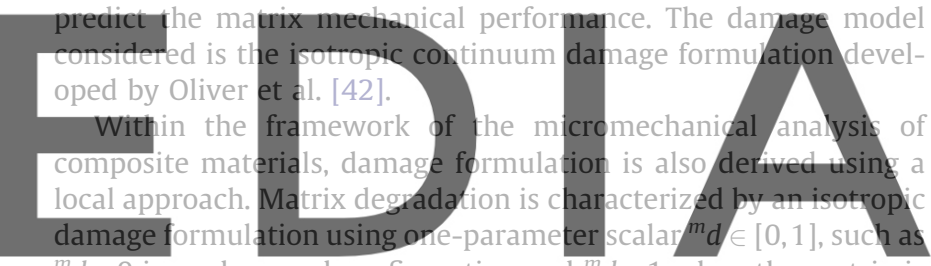
$d=0$ in undamaged configuration and ${ }^{m} d=1$ when the matrix is completely damaged. It is noteworthy that once the materials con-

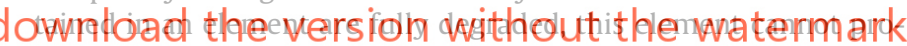
vide further strength to the model and may become excessively distortioned, inducing numerical convergence difficulties forcing the reduccion of the time step. In the simulation procedure proposed below, instead of removing such elements from the finite element mesh, a maximum value that can reach the damage parameters are defined, i.e.: ${ }^{m} d \leqslant 0.99$ and ${ }^{f} d \leqslant 0.99$. This is equivalent to defining a residual stiffness result of the friction between the laminate layers.

Although a scalar damage model does not account for directionality of damage, anisotropic damage effects on the macroscale are taken into account by means of the stacking sequence. The scalar degradation parameter is used to transform the real damaged stress tensor ${ }^{m} \boldsymbol{\sigma}$, into an effective stress tensor ${ }^{m} \boldsymbol{\sigma}_{0}$ corresponding to the matrix undamaged configuration. This relationship can be written as:

${ }^{m} \boldsymbol{\sigma} \equiv\left[\left(1-{ }^{m} d\right) \mathbf{I}\right]:{ }^{m} \boldsymbol{\sigma}_{0}=\left(1-{ }^{m} d\right) \cdot{ }^{m} \boldsymbol{\sigma}_{0}=\left(1-{ }^{m} d\right) \cdot{ }^{m} \mathbb{C}_{0}:{ }^{m} \boldsymbol{\varepsilon}$

A yield function $\mathbb{F}\left({ }^{m} \boldsymbol{\sigma}_{0},{ }^{m} \mathbf{q}\right)$ is used to define an elastic domain $\mathbb{F}<0$ in which no further degradation takes place:

$\mathbb{F}\left({ }^{m} \boldsymbol{\sigma}_{0},{ }^{m} \mathbf{q}\right)=f\left({ }^{m} \boldsymbol{\sigma}_{0}\right)-c\left({ }^{m} d\right) \leqslant 0$ with ${ }^{m} \mathbf{q} \sim{ }^{m} d$

Eq. (8) is written generically because the proposed procedure can use any given yield surface (i.e. Von-Mises, Mohr-Coulomb, etc.). A detailed description of several yield surfaces can be found in [43]. The term $f\left({ }^{m} \sigma_{0}\right)$ depends on the stress tensor and $c\left({ }^{m} d\right)$ corresponds to the evolution of the damage parameter besides defining the threshold of damage growth when $c\left({ }^{m} d\right)={ }^{m} \sigma_{\max }$, defined as a material property. Accordingly damage onset occurs the first time that equivalent stress $f\left({ }^{m} \boldsymbol{\sigma}_{0}\right)=c\left({ }^{m} d\right)$. 
Matrix damage onset is predicted by the norm of the principal stresses by using a different degradation path for tensile and compressive stresses:

$f\left({ }^{m} \boldsymbol{\sigma}_{0}\right)=\rho \cdot\left\|^{m} \boldsymbol{\sigma}_{I}\right\|$

being ${ }^{m} \boldsymbol{\sigma}_{I}$ the principal stress tensor and $\rho$ a weighting function of the tensile and compressive stresses applied to the material. It is defined as:

$\rho=\frac{\sum_{I=1}^{3}\left\langle{ }^{m} \sigma_{I}\right\rangle}{\sum_{I=1}^{3}\left|{ }^{m} \sigma_{I}\right|} \frac{{ }^{m} \sigma_{c}}{{ }^{m} \sigma_{t}}+\left(1-\frac{\sum_{I=1}^{3}\left\langle{ }^{m} \sigma_{I}\right\rangle}{\left.\sum_{I=1}^{3}\right|^{m} \sigma_{I} \mid}\right)$

where ${ }^{m} \sigma_{t}$ and ${ }^{m} \sigma_{c}$ are the tensile and the compressive matrix ultimate strength, respectively, and $\langle\cdot\rangle$ is the Macauley bracket defined as $\langle x\rangle:=\frac{1}{2}(x+|x|)$.

The variation of the damage parameter is obtained by using the damage consistency parameter and the Kuhn-Tucker condition, being possible to explicitly integrate the damage internal variable to obtain:

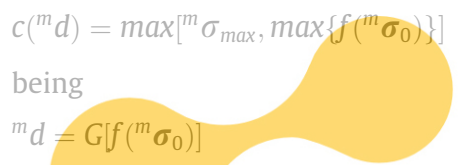

where $\left.G\left[f^{m} \boldsymbol{\sigma}_{0}\right)\right]$ defines the strain-softening evolution of the matrix material. The present work uses an exponential strain-softening law [42], expressed as:

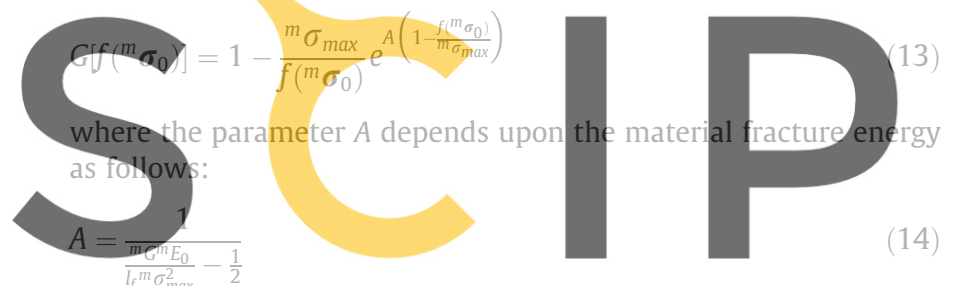

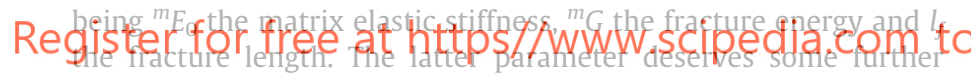
attention to avoid mesh-dependent results: in a finite element implementation the fracture length value must correspond to the smallest value in which the laminate is discretized, i.e. the length represented by an integration point. By doing so, the degradation process becomes mesh independent, as proved in [37].

The above formulation has been implemented into the explicit finite element package ComPack, result of the collaboration between CIMNE and Quantech ATZ. The results of the validation of the formulation are presented in the following sections.

\section{Drop-weight impact test}

The test conditions are decisive on the impact response of a laminate composite. Insofar as possible, impact tests should reproduce the same failure modes induced when the structure is in service. Both failure mechanisms and damage morphology are influenced by intrinsic parameters of the experimental test, such as: mass, shape, diameter, material and the incident velocity of the impactor as well as geometry, layup and the boundary conditions of the coupon. In order to minimize the problems arising from the diversity of test protocols, in this research a test campaign based on standard device has been raised. An ASTM ${ }^{2}$ standard test method for measuring the damage resistance of a fiber-reinforced polymer matrix composite to a drop-weight impact event [44] is followed. Deviations about the standard test are documented and justified. The test method is used to determine the damage

\footnotetext{
${ }^{2}$ American Society for Testing and Materials.
}

resistance of multidirectional composite laminated plates subjected to an out-of-plane and concentrated impact event. The composite damage resistance is quantified in terms of the extent and damage morphology, and the failure modes induced in the laminated specimen.

The test device and the geometry of the coupon are schematised in Fig. 1. It consists of a plate-like fixing support with a rectangular and centered hole cut-out, upon which the sample is placed. The specimen is fixed by four horizontal toggle clamps. ${ }^{3}$ In order to prevent damaging the sample with the force applied by the tip, they are covered with an elastomer. Above the fixing support and perpendicular to it, a cylindrical tube system is installed acting as a guide during the drop-weight. The test procedure consists in releasing the weight from a height which determines the incident kinetic energy. One impact on each specimen is admitted, so that rebound must be intercepted. The impactor is composed of a steel ${ }^{4}$ cylinder of $200 \mathrm{~mm}$ length and $44 \mathrm{~mm}$ diameter, and a smooth hemispherical striker tip with a diameter of $16 \mathrm{~mm}$ and a hardness of $40 \mathrm{HRC}^{5}$ The total mass of the impactor is 2248 gr. This mass value is significantly lower than the $5.5 \pm 0.25 \mathrm{~kg}$ specified in the standard test method. However, the standard also specifies that the minimum height of the drop-weight must be $300 \mathrm{~mm}$. In the case of a minimum level of required incident energy is not reached with these parameters, it specifies that the mass of the impactor may be $2 \pm 0.25 \mathrm{~kg}$.

The experimental program was conducted for a total of 44 monolithic composite plates specimens with a size of $150 \times 100 \times 5.2 \mathrm{~mm}^{3}$. In the manufacturing process unidirectional

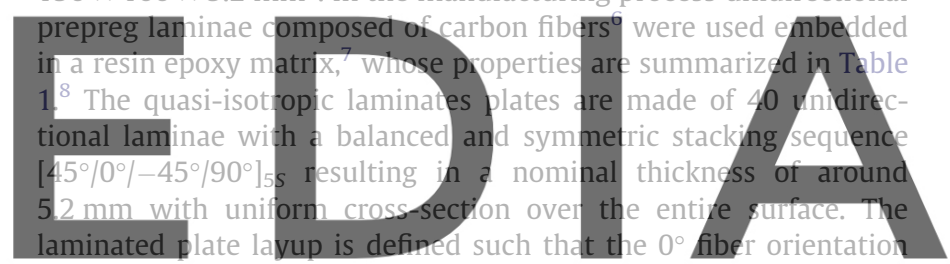
is aligned with the lengthwise dimension. Laminate curing was performed by a standard autoclave procedure. After the curing process,

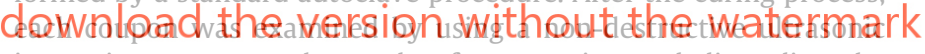
inspection to assess the grade of compaction and discarding the presence of defects, porosities or delaminations.

To evaluate the impact damage resistance of laminated composites plates, specimens were impacted with incident energies levels varying in the range from 6.6 to $70 \mathrm{~J}$, in intervals of $5 \mathrm{~J}$, with a minimum of two and maximum of four specimens for each impact energy. The impact device used to conduct the test makes unfeasible the measurement of contact force during the impact event. ${ }^{9}$ However, it should be pointed out that the interest of this research is not the characterization of the impact event but rather the extent and damage morphology induced. Nevertheless it was considered desirable to measure the contact time and the incident and rebound velocities in order to estimate the energy absorbed during the impact. For this purpose, a strain gauge ${ }^{10}$ attached to the bottom layer and a high-speed camera ${ }^{11}$ were used (see Fig. 1 ). The sample rate of the strain gauge data acquisition system was set at $9600 \mathrm{~Hz}$, which corresponds to a temporal resolution of $104 \mu$ s. The camera

\footnotetext{
3 AMF model 6830-3.

4 UNE F-1150.

5 The hardness of the impactor is less than that specified in the standard $(60$

$62 \mathrm{HRC})$, however no anomalies were observed after impact tests.

${ }^{6}$ Grafil TR30S 3K.

7 Resin epoxy HSC Epikote 4652.

8 Nominal values provided by manufacturers.

${ }^{9}$ It should be noted that on the standard test method the use of velocity and force instrumentation are declared as optional.

10 Strain gauge HBM 1-LY41-6/350. Data recording of the measurements has been performed using MGCplus data acquisition system.

11 MotionBLITZ Cube4.
} 

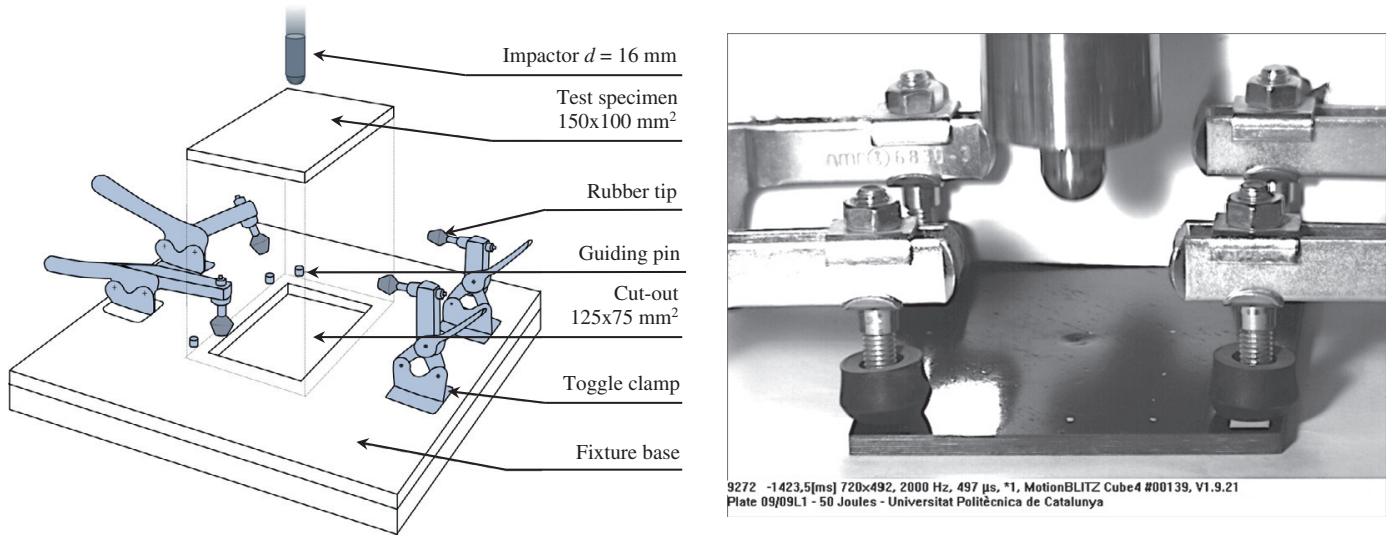

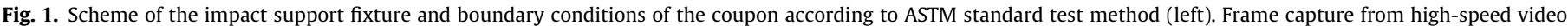
recording corresponding to an instant after contact in the impact test performed (right).

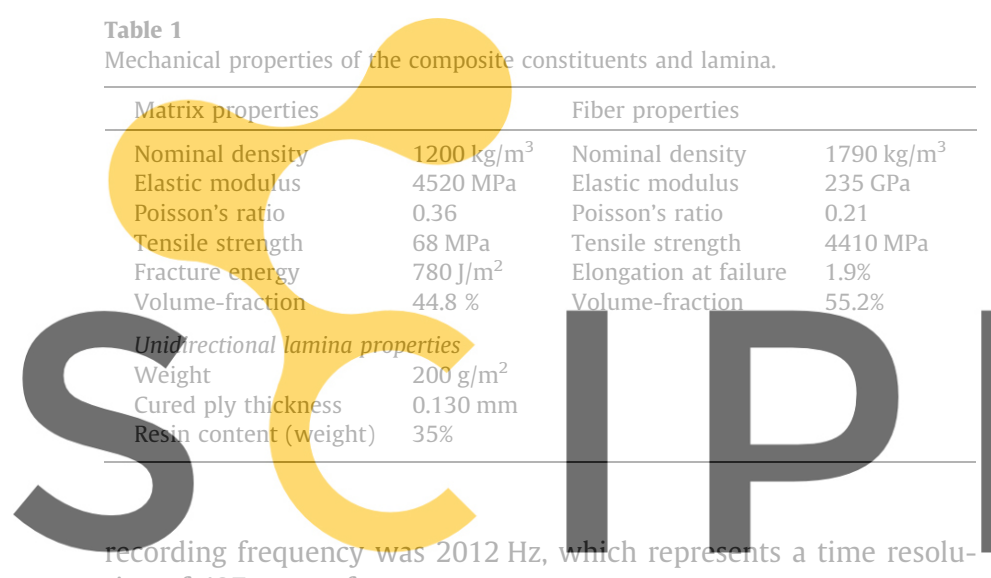
tion of 497 us per frame.

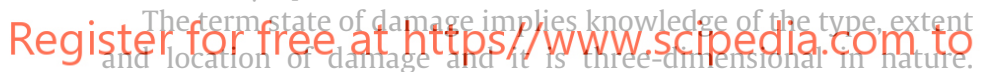

Ultrasonic phased array testing provides a precise through thickness damage information useful for determining the depth, size and distribution of internal delaminations. After the impact event, the extent of damage is measured by using non-destructive ultrasonic inspection equipment. ${ }^{12}$ Using data from B and C-scan, depth and extent of projected area of the induced delaminations are determined.

\section{Numerical simulation of drop-weight impact test}

A numerical study is undertaken to investigate the capability of the proposed numerical formulation when applied to a low-velocity impact problem. The aim of the numerical simulation is to obtain a prediction map of the impact induced internal damage in multidirectional composite laminates plates, as well as assessing the extent of damage over a range of incident energies. A geometrical model, depicted in Fig. 2, was built and appropriate boundary conditions were defined to reproduce the experimental setup described in the above section.

Composite virtual coupon consist of a $75 \times 50 \times 5.2 \mathrm{~mm}^{3}$ rectangular plate which corresponds to one quarter of the overall geometry of the laminate. This simplification assumes a double symmetry in the internal damage distribution to favor a considerable reduction of the computational cost. The virtual coupon is simply supported on the base and is fixed with a toggle clamp, preventing the movement in the out-of-plane direction during

12 OmniScan MX with standard phased array probe $5 \mathrm{MHz}$ linear array 64 elements. the impact of a hemispherical projectile. The impactor diameter is $16 \mathrm{~mm}$, according to the standard test method. The fixture base and the impactor are modeled as solid rigid bodies. Only the holder and the composite virtual coupon are considered deformable parts. The holder and the impactor have a concentrated mass of $0.025 \mathrm{~kg}$ and $0.562 \mathrm{~kg}$ respectively, thus the mass value assigned to the impactor is one quarter of the projectile mass.

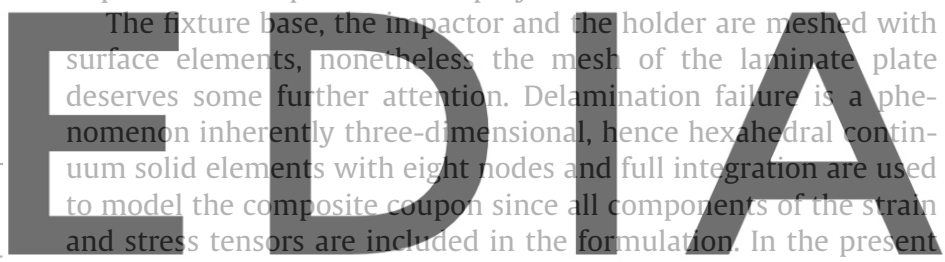
study an initial attempt was made discretizing the composite by placing an element in each layer, however it was not be feasible be-

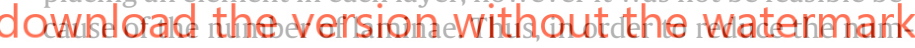
ber of solid elements and consequently the computational cost, several laminae were compacted in a single element. This procedure is of common practice in laminate simulations and it may not compromise the results accuracy as it is shown in [38].

However, although it is possible to collapse some layers in a single element, the through-thickness discretization of the composite virtual coupon plays a major role in the results accuracy (either if the coupon is a composite laminate or if it is a single isotropic material). The lower the number of elements is, the lower resolution of the map of the internal damage will be and the model will exhibit an overly stiff behavior. A larger number of finite elements is preferable but it leads ineluctably to a significantly increase of the computational time required to perform the simulation. In the present work, a finite element mesh of $60 \times 40$ with 12 linear hexahedral elements in the through-thickness direction is selected to achieve an optimal performance, as it provides accurate results with a reasonable computational cost. The resulting mesh has a total of 32,513 nodes. Thus, in each hexahedral element $\frac{10}{3}$ laminae are collapsed. The mesh generated is depicted in Fig. 2. Contacts were defined to set up the set of contact parts pairs, in order to avoid interpenetration between parts.

The composite virtual coupon has a quasi-isotropic stacking sequence $\left[45^{\circ} / 0^{\circ} /-45^{\circ} / 90^{\circ}\right]_{5 s}$ as described in the above section. The mechanical properties of each constituent composite are summarized in Table 1 . The fiber behavior is assumed to be linear up to tensile failure, whereas the matrix damage behavior is modeled following the exponential softening law described in Section 2.

Numerical simulations are carried out for each level of the incident energy conducted on the experimental program. The impactor 


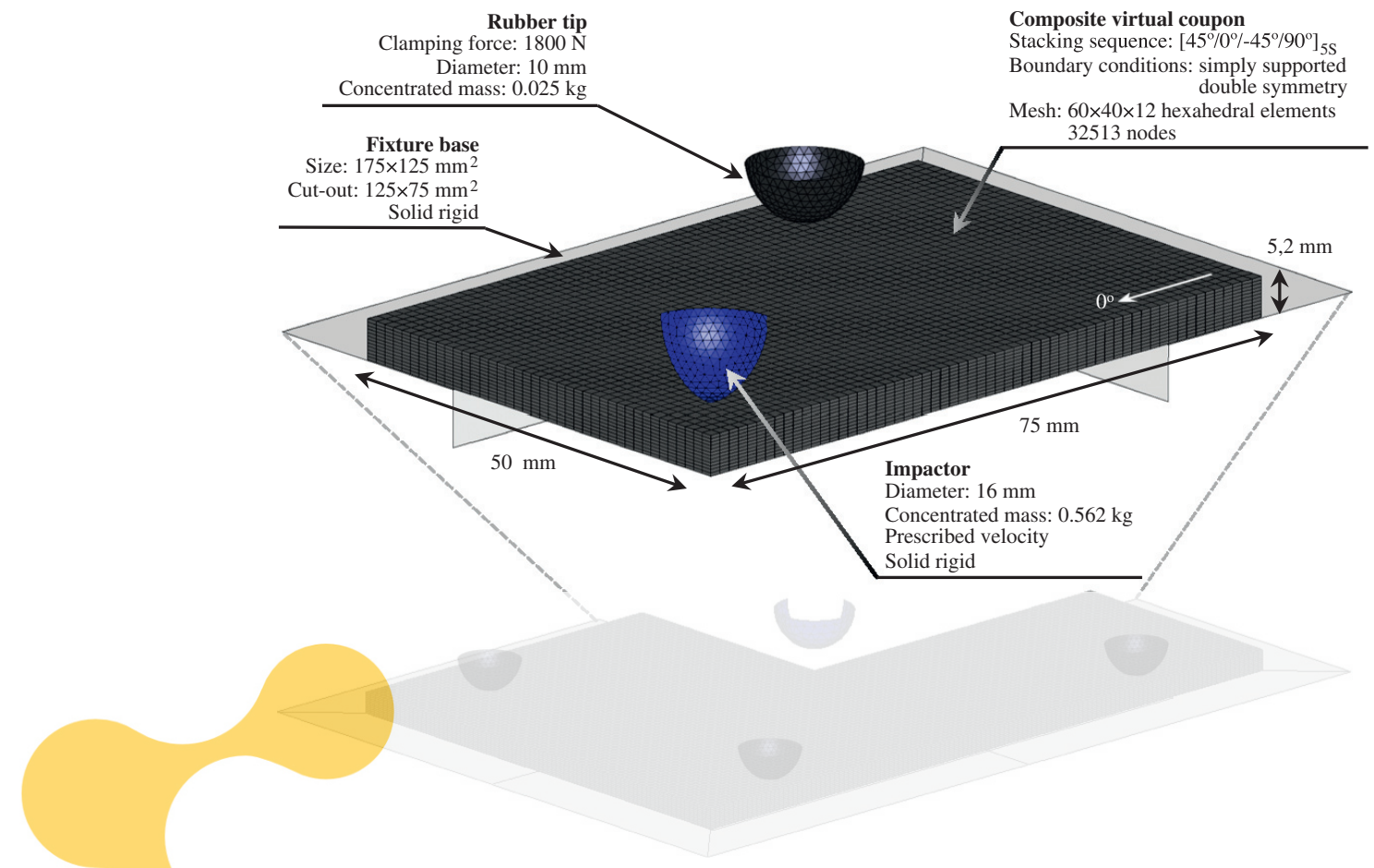

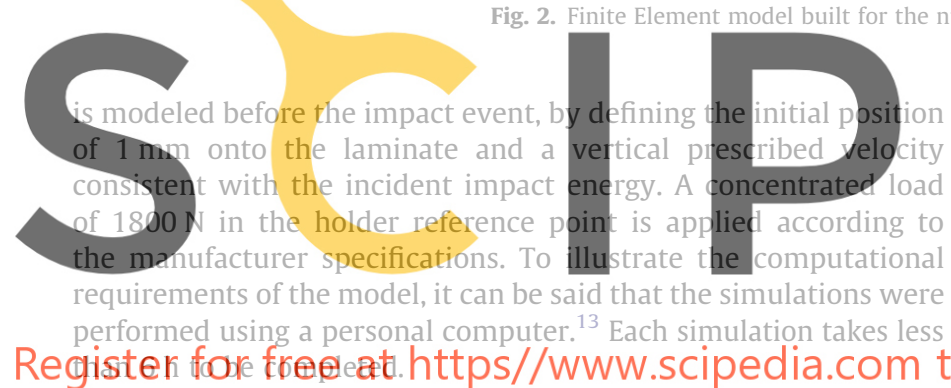

\section{Results and discussion}

The capability of numerical procedure when applied to a lowvelocity impact problem is assessed comparing numerical results with the experimental ones obtained in a test campaign performed. In particular, attention will be given to the impact energy, impact force and the impact induced damage on the laminate.

\subsection{Impact energy}

The impact damage resistance of laminated composites plates is evaluated for impact energies ranging from 6.6 to $70 \mathrm{~J}$. The lower limit is determined by the minimum height condition imposed by the standard test method. The upper limit was defined considering a hypothetical energy level below penetration and perforation thresholds.

During the impact event the impactor's kinetic energy is transferred to the composite plate once contact is made until it reaches in equilibrium. Part of this energy is stored as elastic strain energy and part is absorbed. The absorbed component results from the sum of the contributions given by non-conservative forces and the energy dissipated due to the failure mechanisms. Finally, the stored elastic energy is transferred back to the impactor causing a rebound.

\footnotetext{
13 Intel Core i5 650-3,20 GHz RAM 4 Gb.
}
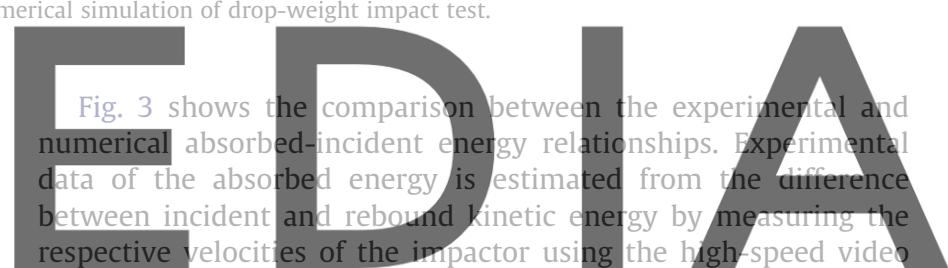

recording. Data plotted represent the mean values with standard deviation bars for each energy level. Numerical data are obtained

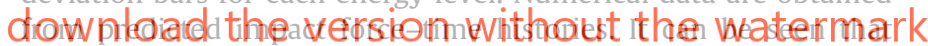
both absorbed energy curves follow a quadratic relationship. These results are consistent with the observations made by Feraboli and Kedward [6]. It should be noticed that the highest incident energy $(70 \mathrm{~J})$ is located below the penetration threshold, represented with a diagonal line corresponding to a null velocity rebound, as intended. Although both curves exhibit similar trends, the deviation from experimental and numerical curves is evident. Difference

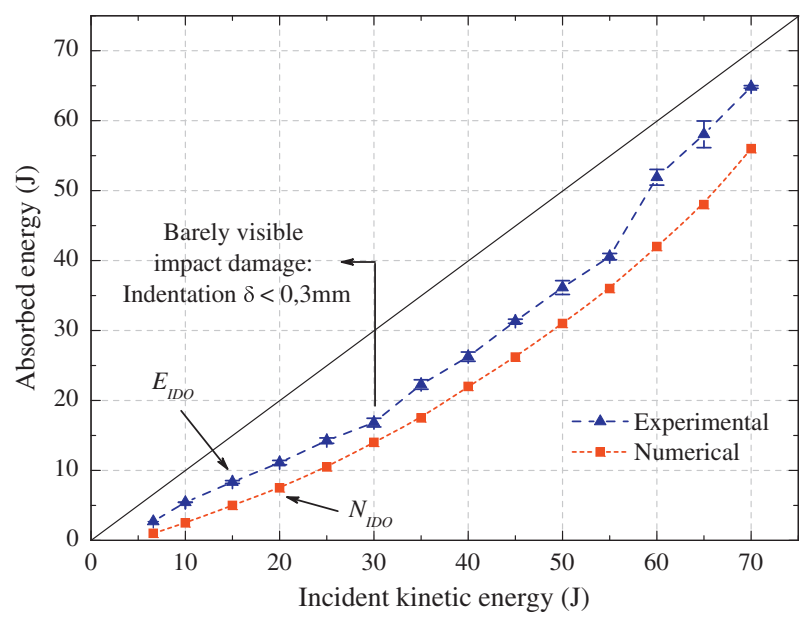

Fig. 3. Experimental and numerical absorbed-incident energy relationships. $E_{I D O}$ and $N_{I D O}$ refer to the experimental and numerical interlaminar damage onset, respectively. 
is mainly due to the fact that on the numerical model, the dissipated energy by friction effects, material damping, permanent indentation, etc. is neglected. The energy dissipation of the model is done through the matrix material and is controlled by the fracture energy parameter $\left({ }^{m} G\right)$. This parameter is provided by the manufacturer and corresponds to the matrix as a bulk material, therefore it cannot account for effects such as friction, etc. In order to take these effects into account, the fracture energy parameter should be calibrated accordingly. The graph presents some indications that refer to the so-called barely visible impact damage (BVID) and the numerical $N_{I D O}$ and experimental $E_{I D O}$ interlaminar damage onset, respectively. The later is determined by the ultrasound inspection results.

\subsection{Impact force}

Fig. 4 shows an example of numerical prediction of the contact force histories for three different impact energies on composite virtual coupon. The prediction curve of the lower (subcritical) impact energy value $\left(E_{I}=10 \mathrm{~J}\right)$ tends to an elastic response, whereas the other two predicted curves show an abrupt drop of the contact force value after the contact initiation. This corresponds to the delamination onset $N_{\text {IDO }}$, whose critical force has been previously shown to be independent of the impact energy [6]. Discrepancy between the peak forces can be attributed to the numerical oscillations. The contact force amplitude variations of the virtual coupon composite curve impacted at $50 \mathrm{~J}$, reveals the onset of fiber breakage when it reaches the maximum contact force (equilibrixum). The contact time depends on the impact higher the impact energy was, the relationship was also observed in the ing a nonlinear trend. It is shown that the contact duration increases due to
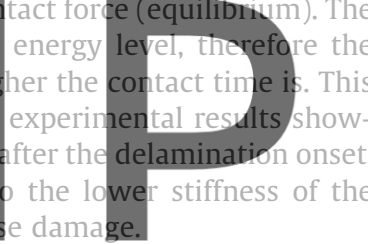

Since the impact device used in the experimental campaign did not allow contact force measurement during the impact event, the

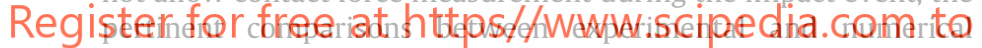
contact force responses are not carried out. Nevertheless, these findings are in good agreement with existing observations resulting from impact tests previously published $[7,11,45]$.

\subsection{Impact induced damage}

One of the main results sought in current research was to obtain the internal delamination in composites due to an impact load. In

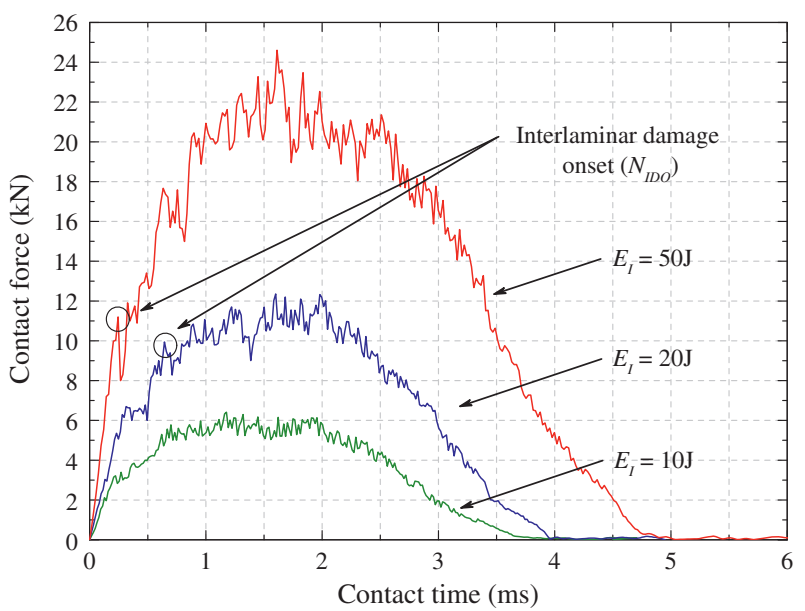

Fig. 4. Numerical prediction of the contact force histories for $10 \mathrm{~J}, 20 \mathrm{~J}$ and $50 \mathrm{~J}$ impact energies on composite virtual coupon. $N_{I D O}$ corresponds to critical force of the delamination onset. the experimental test this damage is obtained by ultrasounds. The validity of the numerical model presented will depend upon if it is capable of reproducing the internal impact induced damage shown by the experimental results.

As an example, Fig. 5 presents the evolution of the throughthickness distribution of damage for a $50 \mathrm{~J}$ simulated impact on the composite virtual coupon. The variable plotted corresponds to the average damage $d_{e}$ on each gauss point of the finite element $e$, which is defined as:

$d_{e}=\frac{1}{V_{e}} \sum_{i=1}^{n}{ }^{L_{i}} k_{e}{ }^{L_{i}} d_{e}\left({ }^{m} d,{ }^{f} d\right)$

being $V_{e}$ the volume of the element, $n$ the number of laminae collapsed in the element, ${ }^{L_{i}} k_{e}$ the volumetric participation of the $i$-lamina and ${ }^{L_{i}} d_{e}$ the damage of the $i$-lamina which is a function of the matrix ${ }^{m} d$ and fiber ${ }^{f \mathrm{~d}}$ damage parameters.
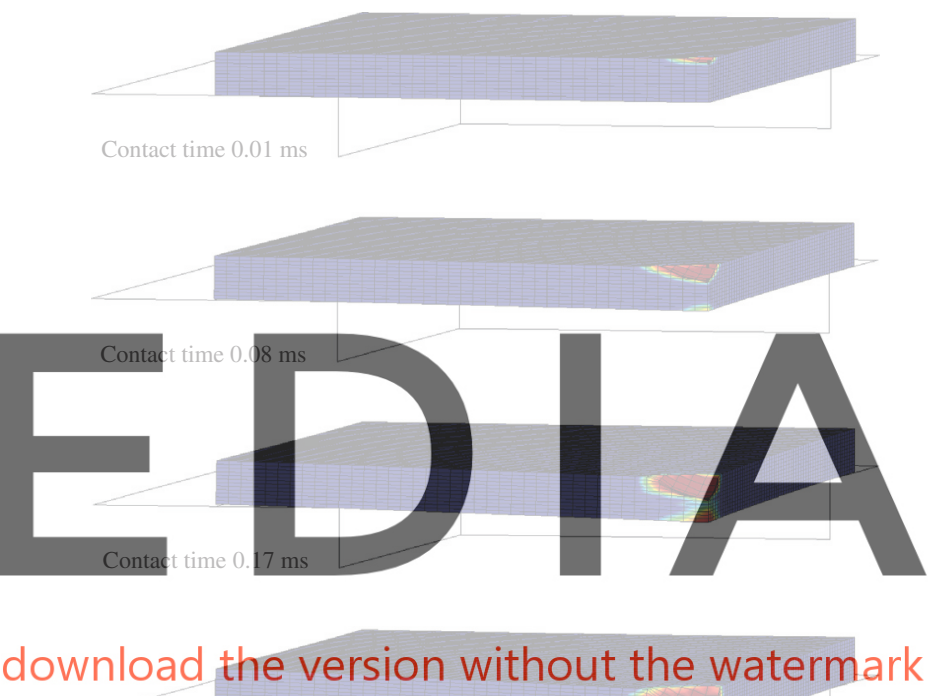

Contact time $0.25 \mathrm{~ms}$
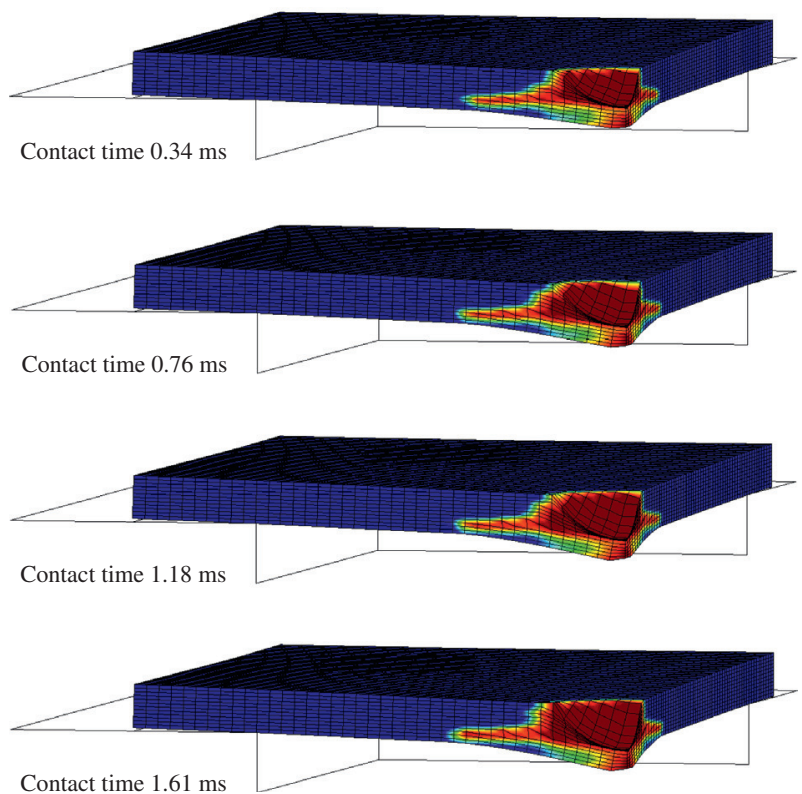

Fig. 5. Quarter section view of the evolution of the through-thickness distribution of damage for a $50 \mathrm{~J}$ simulated impact on $\left[45^{\circ} / 0^{\circ} /-45^{\circ} / 90^{\circ}\right]_{5 s}$ laminate. The variable plotted corresponds to the element average damage. 
The views depicted correspond to a quarter section of the geometry during the loading step. Following contact, damage is induced on the top region at the impact site. As the load level increases, the damage on the bottom region progressively increases due to the bending stress. Starting from contact time $0.25 \mathrm{~ms}$, a growth and propagation of interlaminar damage parameter is observed until the impactor reaches the equilibrium at the point of maximum deflection. During the unloading step, no damage evolution is observed. It should be noticed the sudden increase of interlaminar damage between contact times 0.25 and $0.34 \mathrm{~ms}$. This result is in concordance with the abrupt drop of the contact force depicted in Fig. 4 above.

By observing the kinematic motion described by the element nodes located in the region of interlaminar failure, a state of tensile stress and shear stress is deduced, corresponding to a mixing opening-sliding fracture mode. Moreover, as discussed above, once an element is fully degraded it cannot provide further strength to the model and may become excessively distortioned, inducing numerical convergence difficulties forcing the reduction of the time step. In order to avoid excessive mesh distortion, a residual stiffness remaining in the materials was imposed as set forth in Section 2.2.

Numerical results from the simulation are evaluated by comparing experimental measurements and observations. The main conclusion regarding the experimental results is that the procedure and the test device used for impact damage resistance evaluation, allowed to reproduce the three main failure modes: matrix cracking, delamination and fiber breakage. A cornparison between numerically predicted and experimentally induced internal damage at the end of t in Fig. 6. The extent and spatial distrib was determined by using non-destructive ultr in previously. In general terms

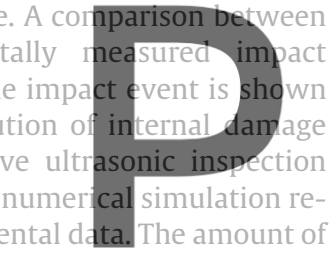

induced damage is more extensive as increases the impact energy, as expected. A conical shape of the internal distribution of damage is observed. It should be noticed that the cross section view of numerical results is depicted symmetrical. Since experimental results confirm the high degree of symmetry of the internal damage distribution, the simplification to one quarter of the overall geometry is considered acceptable to obtain an initial prediction. This simplification is not acceptable when it is necessary to predict the oblong shapes extended along the fibers of each layer.

The ultrasonic image results identify a region (shaded in Fig. 6) located below the delaminations near top surface which has no available experimental data for comparison. This result is due to an inherent limitation of the experimental technique whose effect has been termed the shadow cone. Once ultrasonic wave reaches a delamination, it is reflected back toward the surface, impeding the identification of deeper damages. Numerical predictions indicate a fiber breakage and matrix cracking on the bottom region due to the bending stress, which is consistent with the visual observations made after the experimental tests.

In general, the numerical model is able to predict internal damage induced by low-velocity impact. The extent and distribution of damage is reasonably well predicted. Minor differences in depth of damage arise due to the mesh refinement in the through-thickness direction. It should be noticed that some delaminations are not captured by the numerical model. This is because the simulation uses a continuum phenomenological approach, in which the layers are not defined as a specific element. With this approach damage is

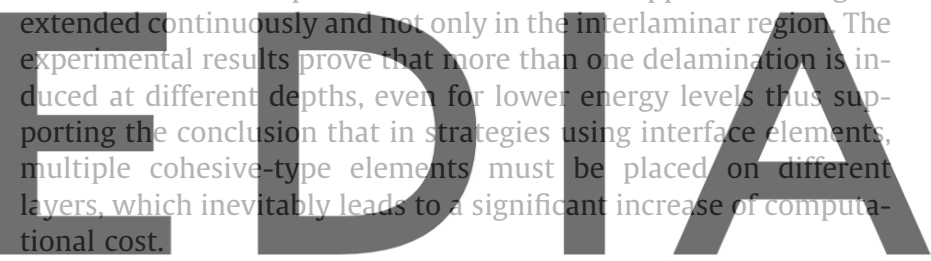

\section{Register for free at https//www.scipedia.com to download the version without the watermark}
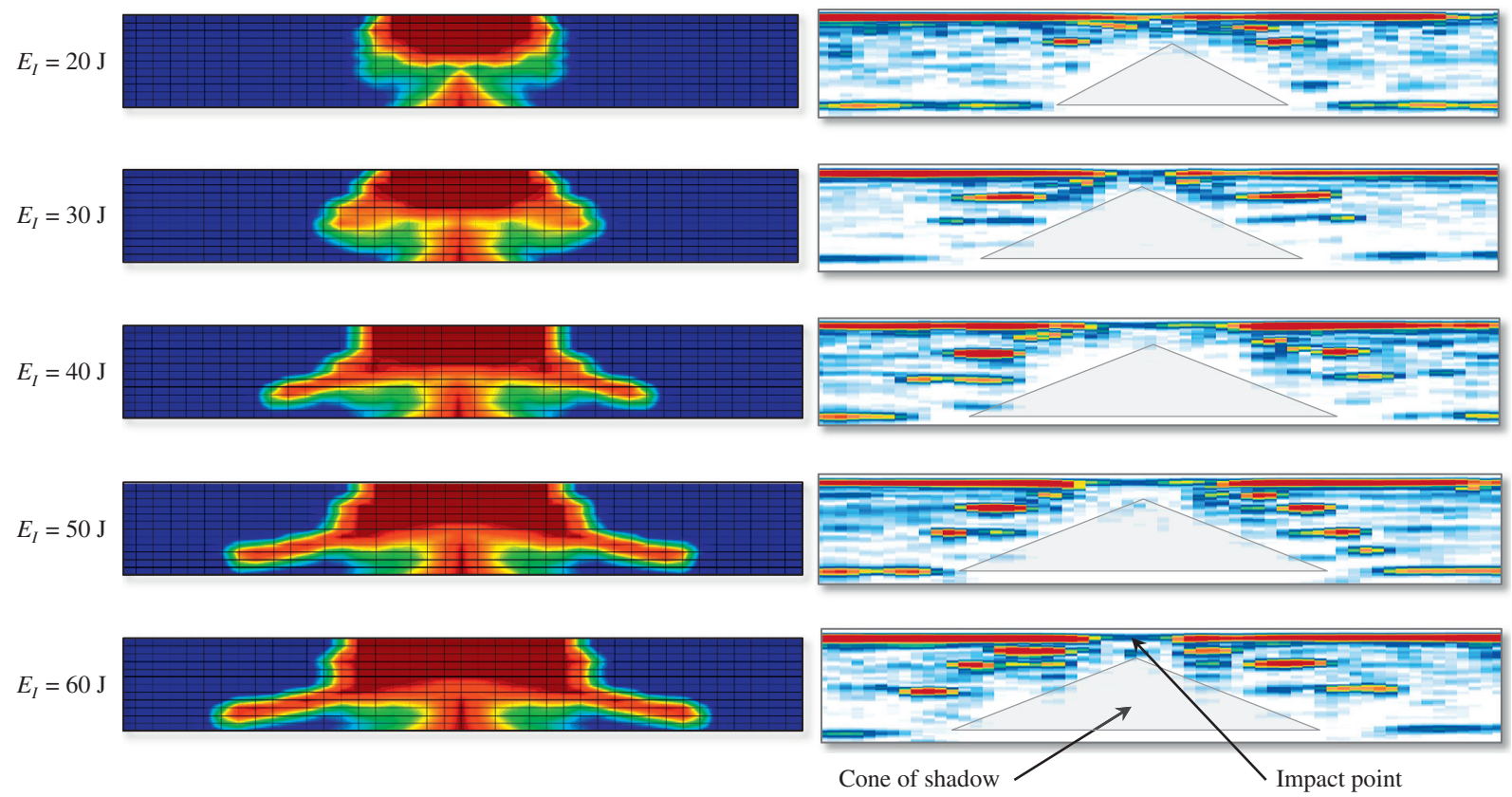

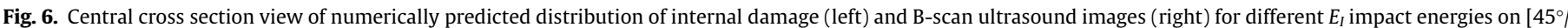

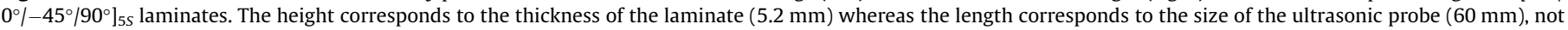
the width of the laminate $(100 \mathrm{~mm})$ 
Quantitative comparison of the damage extension is done in terms of the projected delaminated area. Fig. 7 shows a comparison between numerical prediction of projected delaminated area resulting from the superposition of all internal delaminations and experimental measured induced damage by means of C-scan ultrasonic images, as a function of impact energy. Experimental data plotted represent the mean values with standard deviation bars for each energy level. As Fig. 7 demonstrates, numerical prediction of the projected delaminated area correlates reasonably well with the trend of experimental data, although it tends to underestimate damage extension. When comparing the slopes of the linear curve fitting data across the low energy range - starting from the interlaminar damage onset up to $50 \mathrm{~J}$ - there is an absolute error of $7.2 \%$. The average difference on the predicted delaminated area across the whole energy range is $151 \mathrm{~mm}^{2}$.

It is known that mechanical properties of the composite constituent have great influence on the accuracy of the numerical results, particularly delamination toughness is mainly driven by the matrix fracture energy parameter $\left({ }^{m} G\right)$ as it is shown in [40]. Hence, the delaminated area with increase when matrix fracture energy decreases. And, with increasing matrix fracture energy, more energy is dissipated during delamination growth. In spite of using the properties provided by the manufacturer it is warned about the possible significant variation depending on the laminate cure cycle. According to these observations, it is fair to assume that matrix fracture energy parameter used may not accurately correspond with the parameter of the tested laminates.
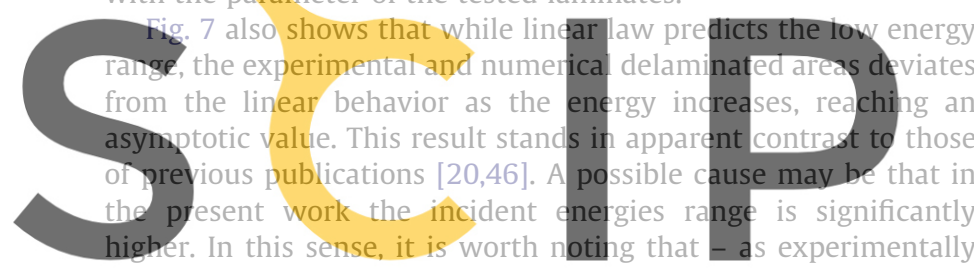

ascertained - the change in slope corresponds with the onset of fiber breakage. Another important aspect to consider is the influence

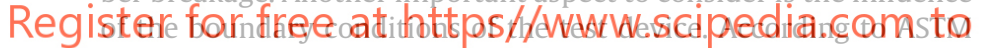
standard test method, the maximum damage diameter should be less than half of the unsupported specimen width (i.e. $37.5 \mathrm{~mm}$ ). C-scan ultrasonic images indicated that this condition is not fulfilled in those laminates that were impacted with energies higher than $50 \mathrm{~J}$, since the width of the damage is approximately $45 \mathrm{~mm}$. This observation is in agreement with prevoius published studies from other authors [47]. At these energy levels it was shown that the stress states generated on contact surfaces of the

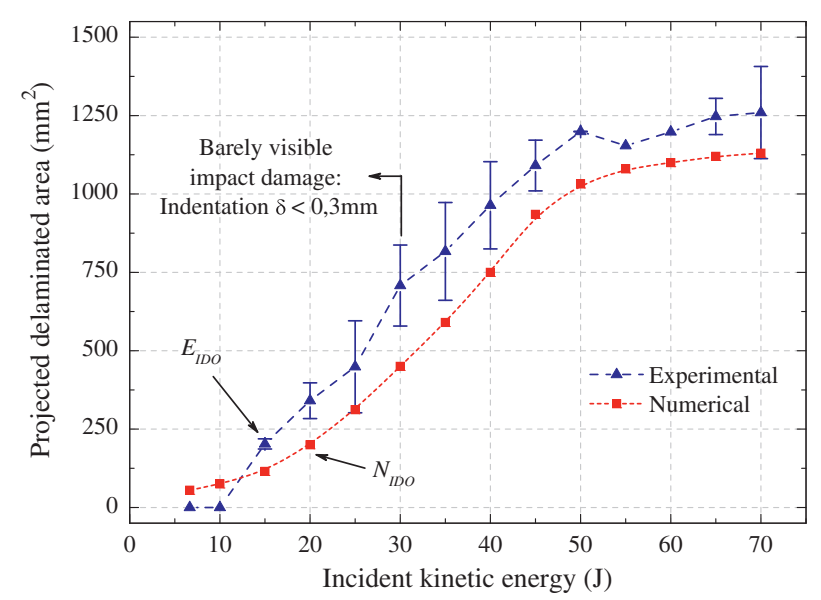

Fig. 7. Experimental and numerical prediction of the projected delaminated area versus incident kinetic energy. $E_{I D O}$ and $N_{I D O}$ refer to the experimental and numerical interlaminar damage onset, respectively. specimen neither induce additional damage nor interacts in the propagation of interlaminar damage.

Focusing on the interlaminar damage onset, the numerical simulation indicated that delamination threshold energy level is roughly estimated at $20 \mathrm{~J}$, which is in line with the delamination onset observed experimentally as shown in Fig. 6. Projected area values below this energy level correspond to surface damages, not delaminations. Interlaminar damage onset is then considered when the internal extent of damage is greater than the outer extent of damage. A closer examination shows that the numerical model slightly overpredicted the interlaminar damage onset. Besides fracture energy, another cause may be the mesh resolution since it is not enough to discern between matrix damage and interlaminar damage.

\section{Conclusions}

A new procedure based on a continuum mechanics approach used to perform numerical predictions of impact induced internal damage in composite laminates has been presented and discussed in this paper. The procedure simulates the composite performance by using a proposed micro-mechanical approach named matrixreinforced mixing theory (MR RoM), in which is consider that matrix is only reinforced in fiber direction and that transverse composite behavior is only governed by the matrix. MR RoM is a simplified version of the serial/parallel mixing theory that does

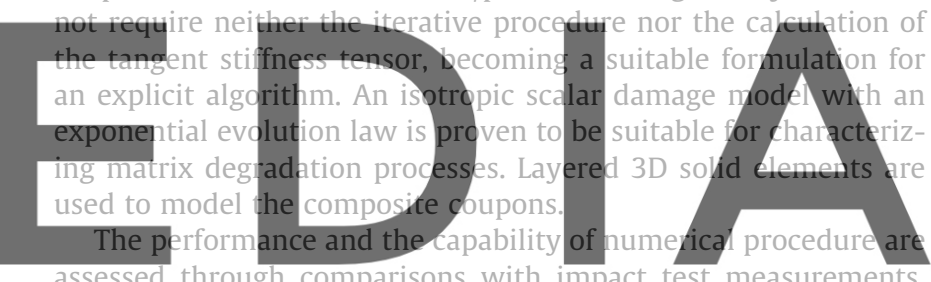
Results and discussions regarding the absorbed-incident energy

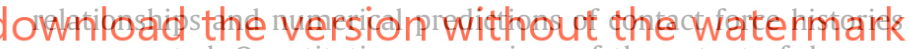
are presented. Quantitative comparison of the extent of damage is done in terms of the projected delaminated area. In general, results are in agreement with experimental data regarding the delamination onset and the internal spatial distribution of induced damage. It is found that numerical model slightly overpredicts the interlaminar damage onset and tends to underestimate the extent of damage. The matrix fracture energy parameter and mesh refinement in the through-thickness direction highly influences the accuracy of the numerical results.

Despite these limitations, the proposed procedure proves to be capable of successfully simulate the composite structural performance when applied to a low-velocity impact problem, and particularly to predict the phenomenon of initiation and propagation of interlaminar damage with no need to formulate interface elements that predefine the interlaminar crack path, resulting in a computationally less demanding formulation as compared to previously published formulations and procedures. The implementation of the MR RoM into a finite element code and its capabilities proves to be promising as a numerical tool to perform large scale impact simulations of composite structures.

\section{Acknowledgements}

This work has been supported by the Spanish Ministry of Science and Innovation through Grant No. UNPC08-4E-007, RECOMP Project, Ref. BIA2005-06952 and DELCOM Project Ref. MAT200802232/MAT., by the Spanish Ministry of Public Works (Project "Retrofitting and reinforcement of reinforced concrete structures with composite materials. Numerical and experimental 
developments applied to joint of bars and composites anchorage proposal") by AIRBUS (Spain) through the Contract No. PBSO-1306 FEMCOM and ACCIONA Infraestructuras (Spain) through the Project SPHERA. All these support are gratefully acknowledged.

\section{References}

[1] Reid S, Zho G. Impact behaviour of fibre-reinforced composite materials and structures. CRC Press; 2000.

[2] Varas D, Artero-Guerrero J, Pernas-Sánchez J, López-Puente J. Analysis of high velocity impacts of steel cylinders on thin carbon/epoxy woven laminates. Compos Struct 2013;95:623-9.

[3] Artero-Guerrero J, Pernas-Sánchez J, Varas D, López-Puente J. Numerical analysis of CFRP fluid-filled tubes subjected to high-velocity impact. Compos Struct 2013;96:286-97.

[4] Hodgkinson J. Mechanical testing of advanced fibre composites. CRC Press; 2000.

[5] Abrate S. Modeling of impacts on composite structures. Compos Struct 2001;51:129-38.

[6] Feraboli P, Kedward KT. A new composite structure impact performance assessment program. Compos Sci Technol 2006;66:1336-47.

[7] Lopes CS, Seresta O, Coquet Y, Gnrdal Z, Camanho PP, Thuis B. Low-velocity impact damage on dispersed stacking sequence laminates. Part I: experiments. Compos Sci Technol 2009;69:926-36.

[8] Tita V, de Carvalho J, Vandepitte D. Failure analysis of low velocity impact on thin composite laminates: experimental and numerical approaches. Compos Struct 2008;83:413-28.

[9] Aktaş M, Atas C, Issten BM, Karakuzu R. An experimental investigation of the impact response of composite laminates. Compos Struct 2009;87:307-13.

[10] Li C, Hu N, Yin Y, Sekine H, Fukunaga H. Low-velocity impact-induced damage of continuous fiber-reinforced composite laminates. Part I. An FEM numerical model. Composites Part A 2002;33:1055-62.

[11] Gonzalez EV, Maimf P, Camanho PP, Turon A, Mayugo JA. Simulation of drop-

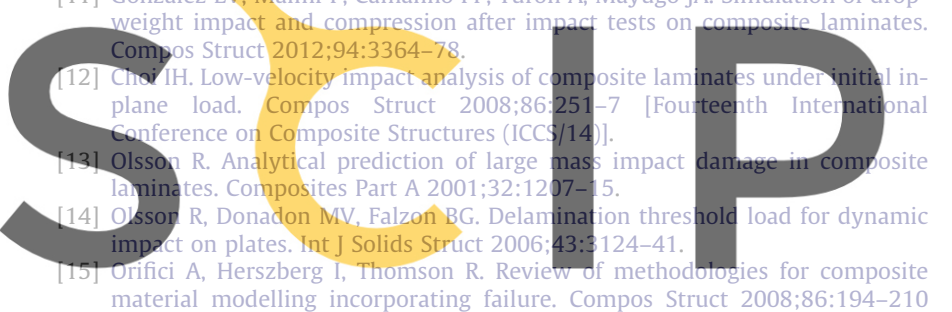
material modelling incorporating failure. Compos Struct 2008;86:194-210 [Fourteenth International Conference on Composite Structures (ICCS/14)].

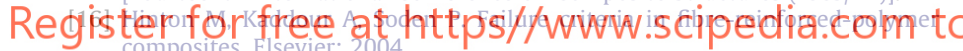

[17] Donadon MV, Iannucci L, Falzon BG, Hodgkinson JM, de Almeida SFM. A progressive failure model for composite laminates subjected to low velocity impact damage. Comput Struct 2008;86:1232-52.

[18] Oller S, Car E, Lubliner J. Definition of a general implicit orthotropic yield criterion. Comput Method Appl M 2003;192:895-912.

[19] Wang J, Qiao P. On the energy release rate and mode mix of delaminated shear deformable composite plates. Int J Solids Struct 2004;41:2757-79.

[20] Zou Z, Reid S, Li S, Soden P. Application of a delamination model to laminated composite structures. Compos Struct 2002;56:375-89.

[21] Shen F, Lee K, Tay T. Modeling delamination growth in laminated composites. Compos Sci Technol 2001;61:1239-51.

[22] Car E, Oller S, Oñate E. A large strain plasticity model for anisotropic materials - composite material application. Int J Plast 2001;17:1437-63.

[23] Williams KV, Vaziri R, Poursartip A. A physically based continuum damage mechanics model for thin laminated composite structures. Int J Solids Struct 2003;40:2267-300.

[24] Iannucci L. Progressive failure modelling of woven carbon composite under impact. Int J Impact Eng 2006;32:1013-43.
[25] Maimf P, Camanho P, Mayugo J, Davila C. A continuum damage model for composite laminates: Part I: constitutive model. Mech Mater 2007;39:897-908.

[26] Pagano N, Schoeppner G. Delamination of polymer matrix composites: problems and assessment. In: Kelly A, Zweben C, editors. Comprehensive composite materials. Oxford: Pergamon; 2000. p. 433-528.

[27] Krueger R. Virtual crack closure technique: history, approach and applications. Appl Mech Rev 2004;57:109-43.

[28] Ortiz M, Pandolfi A. Finite-deformation irreversible cohesive elements for three-dimensional crack propagation analysis. Int J Numer Methods Eng 1999;44:1267-82.

[29] Turon A, Camanho P, Costa J, Davila C. A damage model for the simulation of delamination in advanced composites under variable-mode loading. Mech Mater 2006;38:1072-89.

[30] Turon A, Camanho P, Costa J, Renart J. Accurate simulation of delamination growth under mixed-mode loading using cohesive elements: definition of interlaminar strengths and elastic stiffness. Compos Struct 2010;92:1857-64.

[31] Yang Q Cox B. Cohesive models for damage evolution in laminated composites. Int J Fract 2005;133:107-37.

[32] Camanho P, Davila C, De Moura M. Numerical simulation of mixed-mode progressive delamination in composite materials. J Compos Mater 2003;37:1415-38.

[33] Balzani C, Wagner W. An interface element for the simulation of delamination in unidirectional fiber-reinforced composite laminates. Eng Fract Mech 2008; 75:2597-615.

[34] Borg R, Nilsson L, Simonsson K. Modeling of delamination using a discretized cohesive zone and damage formulation. Compos Sci Technol 2002;62:1299-314.

[35] Lopes C, Camanho P, Gnrdal Z, Maimf P, Gonzalez E. Low-velocity impact damage on dispersed stacking sequence laminates. Part II: numerical simulations. Compos Sci Technol 2009;69:937-47.

[36] Turon A, Davila C, Camanho P, Costa J. An engineering solution for mesh size effects in the simulation of delamination using cohesive zone models. Eng Fract Mech 2007;74:1665-82.

[37] Martinez X, Oller S. Numerical simulation of matrix reinforced composite

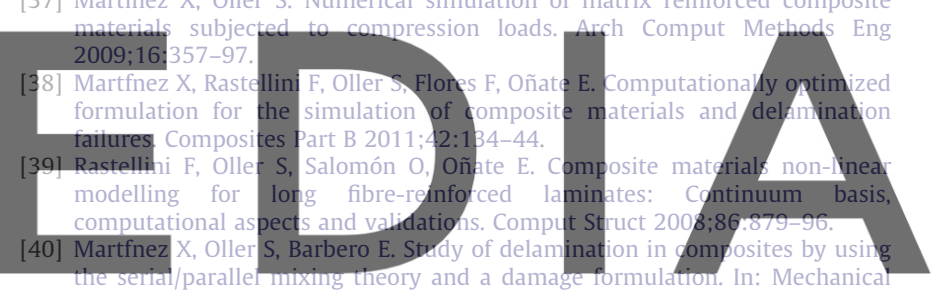
response of composites. Computational methods in applied sciences, vol 10. Netherlands: Springer; 2008. p. 119-40

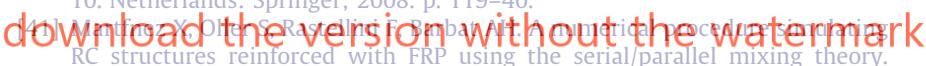
Comput Struct 2008;86:1604-18.

[42] Oliver J, Cervera M, Oller S. Isotropic damage models and smeared crack anaiysis of concrete. in: Proceedings of SCI-C 1990; 1990. p. 945-958.

[43] Lubliner J. Plasticity theory, dover books on engineering series. Dover Publications; 2008.

[44] ASTM-D7136, Standard test method for measuring the damage resistance of a fibre-reinforced polymer matrix composite to a drop-weight impact event. Tech. rep. West Conshohocken (PA, USA): American Society for Testing and Materials (ASTM); 2007.

[45] Shi Y, Swait T, Soutis C. Modelling damage evolution in composite laminates subjected to low velocity impact. Compos Struct 2012;94:2902-13.

[46] Li C, Hu N, Cheng J, Fukunaga H, Sekine H. Low-velocity impact-induced damage of continuous fiber-reinforced composite laminates. Part II. Verification and numerical investigation. Compos Part A: Appl Sci Manuf 2002;33:1063-72.

[47] Gonzalez E, Maimf P, Camanho P, Lopes C, Blanco N. Effects of ply clustering in laminated composite plates under low-velocity impact loading. Compos Sci Technol 2011;71:805-17. 